ELECTRONIC LETTER

\title{
Subcellular localisation of marenostrin/pyrin isoforms carrying the most common mutations involved in familial Mediterranean fever in the presence or absence of its binding partner ASC
}

\author{
C Cazeneuve, S Papin, I Jéru, P Duquesnoy, S Amselem
}

J Med Genet 2003;41:e24 (http://www.jmedgenet.com/cgi/content/full/41/3/e24). doi: 10.1136/jmg.2003.011601

$\mathrm{F}$ amilial Mediterranean fever (FMF) is an autosomal recessive disorder characterised by recurrent episodes of fever and serosal inflammation, which is common in Armenian, Turkish, Arab, and Sephardic Jewish populations. For many years, its diagnosis was one of exclusion, based solely on clinical criteria. However, this difficult situation was changed in 1997 by the identification of the causative gene, $M E F V$, a discovery that has opened the possibility of facilitating the diagnosis of this disease. Indeed, as shown in several population based studies, the analysis of $M E F V$, mutations of which underlie $\mathrm{FMF}^{12}$, has provided the first objective test of diagnostic value. However, although more than $40 \mathrm{MEFV}$ mutations have been identified, ${ }^{3}$ no unambiguous mutations, such as gross gene rearrangements, nonsense, frameshift, or splice mutations, have been reported, except a single report of one $M E F V$ allele carrying a nonsense mutation in the last coding exon. ${ }^{4}$ The most frequent mutations are conservative missense variations located in the $3^{\prime}$ end of the gene, a sequence that encodes a so-called B30.2/SPRY domain of unknown function. As no assay has emerged to evaluate the functional consequences of the missense mutations identified in patients with FMF, these sequence variations are considered as disease causing mutations only on the basis of their high frequency in patients.

Although the first $M E F V$ mutations were identified 6 years ago, the pathophysiology of FMF remains unclear; and the physiological role of the marenostrin/pyrin $(\mathrm{M} / \mathrm{P})$ protein encoded by $M E F V$ is still to be elucidated, even though several lines of indirect evidence strongly suggest the involvement of $\mathrm{M} / \mathrm{P}$ in both inflammatory and apoptotic pathways. ${ }^{5-8} \mathrm{M} / \mathrm{P}$ has been shown to belong to an expanding family of molecules containing a protein-protein interaction domain, called a PYD domain. $.^{79} 10 \mathrm{In} \mathrm{M} / \mathrm{P}$, this domain, which is encoded by the first $M E F V$ exon, is common to several M/P isoforms generated through alternative splicing, ${ }^{11}{ }^{12}$ such as $\mathrm{M} / \mathrm{P}-\mathrm{fl}$, which corresponds to the full length protein encoded by the $10 M E F V$ exons, and other shorter isoforms including $\mathrm{M} / \mathrm{P}-\mathrm{d} 2$, which results from an in frame splice removal of the second $M E F V$ exon. ${ }^{11}$ As shown in the first expression studies, the subcellular localisation of $\mathrm{M} / \mathrm{P}-\mathrm{fl}$ is exclusively cytoplasmic, ${ }^{11} 14$ whereas $\mathrm{M} / \mathrm{P}-\mathrm{d} 2$ is concentrated mainly in the nucleus. ${ }^{11}$ More recent studies have shown, however, that the $\mathrm{M} / \mathrm{P}-\mathrm{fl}$ localisation pattern depends on the presence of ASC (apoptosis associated speck-like protein containing a caspase recruitment domain (CARD)), an $\mathrm{M} / \mathrm{P}$ interacting protein containing an N-terminal PYD domain. ${ }^{75}$ This interaction, which was shown to occur through the PYD domains of M/P$\mathrm{fl}$ and $\mathrm{ASC},{ }^{7}$ results in the formation of cytoplasmic hollow centred aggregates called specks, in which there is a colocalisation of $\mathrm{M} / \mathrm{P}-\mathrm{fl}$ with ASC. Although the functional

\section{Key points}

- The diagnosis of familial Mediterranean fever (FMF), an autosomal recessive auto-inflammatory disorder, was one of exclusion until MEFV gene analysis provided the first objective diagnostic test. However, no assay has emerged to evaluate the functional consequences of the underlying missense mutations that account for most mutant alleles.

- Marenostrin/pyrin (M/P), the protein encoded by MEFV, exists in several isoforms, including M/P-fl and the M/P-d2 isoform that results from alternative splicing of exon 2. The subcellular localisation of normal $M / P$ depends on this splicing event and on ASC, an M/P interacting protein; M/P-fl and M/P-d2 are located in the cytoplasm and the nucleus, respectively, whereas coexpression of M/P-fl with ASC results in the formation of aggregates, termed specks, in which both proteins colocalise.

- To assess the effect of the most frequent MEFV mutations on the subcellular localisation of $M / P$ isoforms, we expressed normal or mutated M/P-fl or -d2 in HeLa cells, in the absence or presence of ASC.

- Cells expressing mutated M/P-fl or -d2 exhibited a subcellular localisation pattern similar to that of corresponding wild type isoforms. The coexpression of each mutated M/P-fl protein with ASC did result in the formation of specks in which the two proteins colocalised. Similar cytoplasmic specks, with no nuclear labelling, were observed when wild type or mutated M/P-d2 isoforms were expressed with ASC, demonstrating that ASC is able to interact with M/P-d2 and dramatically modifies M/P-d2 cell compartmentalisation.

- These findings indicate that the functional consequences of the FMF associated mutations are probably unrelated to a mislocalisation of the mutant $M / P$, even in the presence of its binding partner, ASC.

Abbreviations: ASC, apoptosis associated speck-like protein containing a caspase recruitment domain; BSA, bovine serum albumin; DAPI, $4^{\prime}, 6^{\prime}-$ diamidino-2-phenylindole; FMF, familial Mediterranean fever; $M / P$, marenostrin/pyrin; NGS, normal goat serum 
significance of these specks is unclear, their role in apoptosis regulation has been suggested, with ASC functioning as an adaptor between PYD and CARD containing proteins. ${ }^{6}$

To gain insights into the pathophysiology of FMF, we investigated the effect of the most frequent $M E F V$ mutations and that of one rare mutation located in the PYD domain of $\mathrm{M} / \mathrm{P}$ on the subcellular localisation of $\mathrm{M} / \mathrm{P}-\mathrm{fl}$ and $-\mathrm{d} 2$ isoforms, in both the absence and the presence of ASC.

\section{METHODS}

\section{Plasmid constructs}

The cDNAs coding for $M E F V-f l$ and $M E F V-d 2$ were obtained by digestion of the previously described pMEFV-fl-GFP and pMEFV-d2-GFP expression vectors ${ }^{11}$ by EcoRI and SalI. The resulting products corresponding to $M E F V-f l$ and $M E F V-d 2$ were then cloned into the pEGFP-N3 expression vector (Clontech), which had been previously modified by mutagenesis in order to inverse the SalI and EcoRI restriction sites of the polylinker. The resulting plasmids were named pEGFPMEFV-fl and pEGFP-MEFV-d2. The M694V, M694I, M680I, V726A, E148Q, and A89T mutations were then introduced into pEGFP-MEFV-fl and pEGFP-MEFV-d2 using the QuickChange site directed mutagenesis system (Stratagene). The cDNA encoding human ASC was generated from total leukocyte RNA, and amplified with forward primer 5' -CCCGCGTCGACGCAGCGGCCGGGGATCCTGGAGCC-3' and reverse primer 5'-GCCCAGAATTCCTCAGCTCCGCTCCAGGTCCTC-3'. The resulting RT-PCR product was subsequently cloned into pcDNA3.1/V5-His-TOPO (Invitrogen), in frame with the V5 epitope, to generate the pASC-V5 expression plasmid.

\section{Cell growth, transfection, and immunofluorescence analysis}

HeLa cells were maintained in Dulbecco modified Eagle's medium (Invitrogen) supplemented with $10 \%$ fetal calf serum. HeLa cells $\left(3 \times 10^{5}\right)$ were cultured for 24 hours on glass coverslips in 6 well culture plates, and transfected using $500 \mathrm{ng}$ of the wild type or mutated $M E F V$ constructs and/or $500 \mathrm{ng}$ of pASC-V5, according to the manufacturer's instructions (Lipofectamine-Invitrogen). Eighteen hours after transfection, cells were fixed in $4 \%(\mathrm{v} / \mathrm{v})$ paraformaldehyde and permeabilised with PBS containing $0.1 \%$ Triton (PBS-T). Cells expressing ASC-V5 were then stained as follows. Saturation was performed in PBS-T with $30 \mathrm{mg} / \mathrm{ml}$ bovine serum albumin (BSA) and 5\% normal goat serum (NGS). The coverslips were then incubated in PBS-T containing $10 \mathrm{mg} / \mathrm{ml}$ BSA and 5\% NGS, and a primary antibody directed against the V5 epitope (Invitrogen). After three washes in PBS-T, a 1 hour incubation with a Cy3 tagged secondary antibody (Sigma) in PBS-T with $10 \mathrm{mg} / \mathrm{ml} \mathrm{BSA}$ and 5\% NGS was performed. Cells were then washed three times in PBS-T. DNA was stained with $4^{\prime}, 6^{\prime}$-diamidino-2-phenylindole (DAPI), and cells were examined with a Leica DMR fluorescence microscope.

\section{RESULTS AND DISCUSSION}

In order to assess the effect of the most frequent FMF associated mutations on the subcellular localisation of the M/ $\mathrm{P}$ protein, we first transiently expressed the wild type or mutated M/P-fl or - $\mathrm{d} 2$ proteins in the absence of ASC. HeLa cells expressing the wild type M/P-fl-GFP protein displayed a cytoplasmic labelling very similar to that previously reported in $\mathrm{CHO}$ cells $^{11}$ (fig 1A). Consistent with our previous study performed in CHO cells, ${ }^{11}$ we found that M/P-d2-GFP localised mainly to the nucleus (fig $1 \mathrm{~B}$ ); however, in some cells, the labelling was homogeneously spread all over the cell (data not shown). Most importantly, cells expressing the mutated M/P-fl or - $\mathrm{d} 2$ proteins (that is, carrying the M694V, V726A, M680I, or A89T mutation) showed a subcellular
A
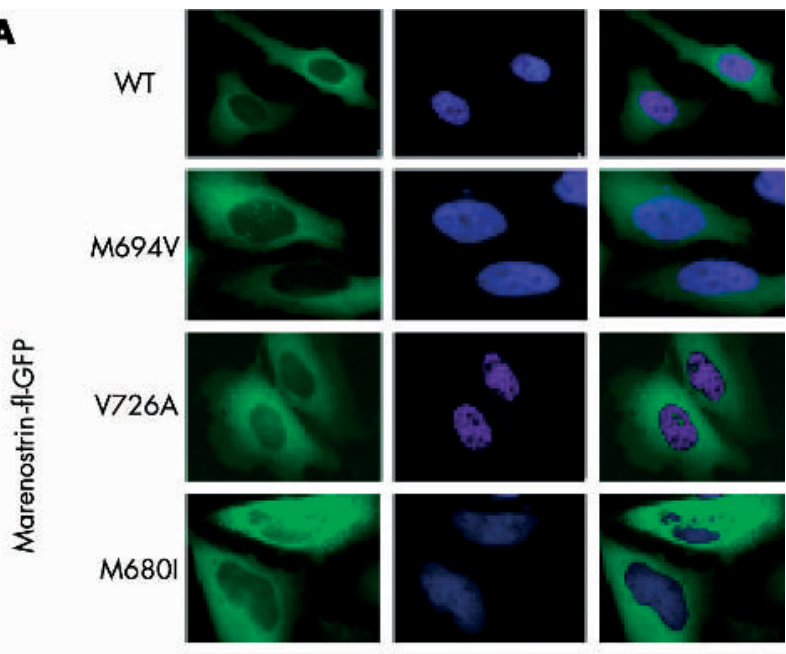

A89T
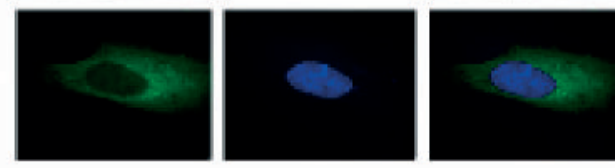

B
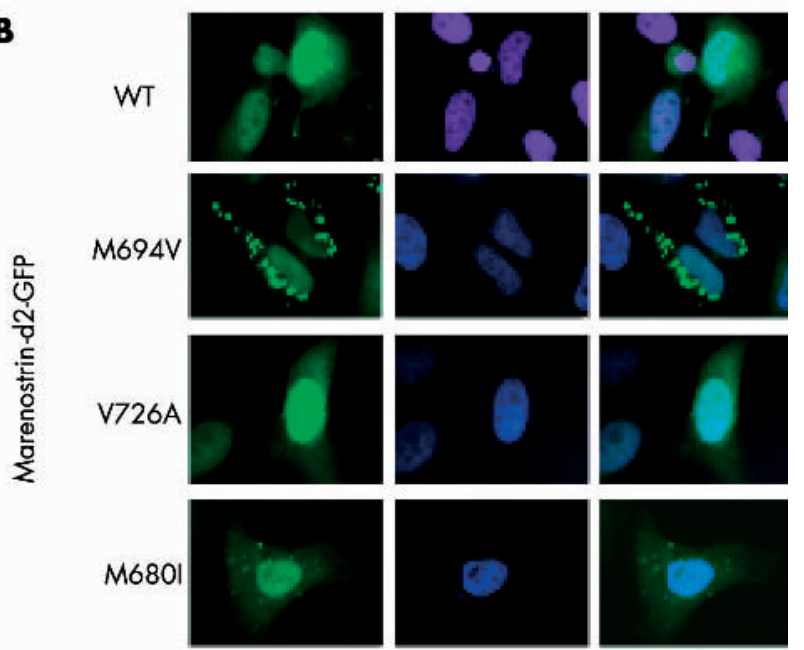

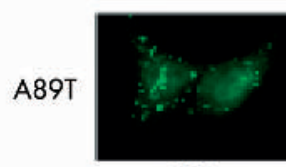

GFP

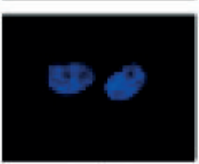

DAPI

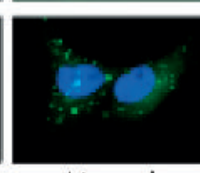

Merged
Figure 1 Subcellular localisation of wild type or mutated M/P-fl and $-d 2$ in HeLa cells, transfected with wild type or mutated pEGFP-MEFV-fl (A) or pEGFP-MEFV-d2 (B) expression vectors. The M/P protein was visualised directly with GFP fluorescence (left). Corresponding DAPI staining and merged images are shown in the middle and on the right, respectively. The data shown are representative of several transient transfections of each construct. Cells expressing low levels of recombinant proteins are shown (see text).

localisation pattern that did not differ from that of the corresponding wild type isoforms (fig 1A,B). Similar results were obtained with the $\mathrm{M} / \mathrm{P}-\mathrm{fl}$ and $-\mathrm{d} 2$ isoforms carrying the M694I mutation, or with M/P-fl carrying the E148Q mutation located in exon 2 (data not shown). In addition, whatever the $\mathrm{M} / \mathrm{P}-\mathrm{fl}$ construct used in transfection assays, we observed that cells expressing a low level of recombinant proteins exhibited a diffuse labelling homogeneously distributed over the entire cytoplasm (fig 1A), whereas high expression levels of $\mathrm{M} / \mathrm{P}$ resulted in the formation of fluorescent punctuations or large perinuclear aggregates over a faint and homogeneous cytoplasmic labelling (data not shown). The two latter 
A

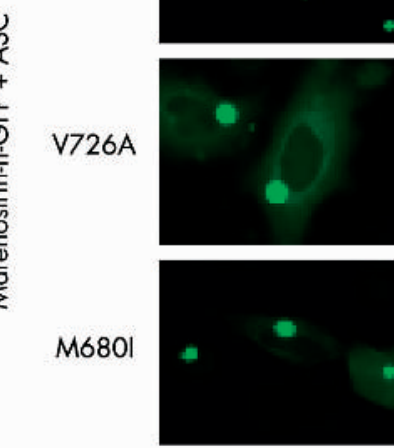

A89T

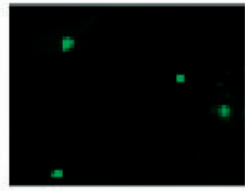

B

WT
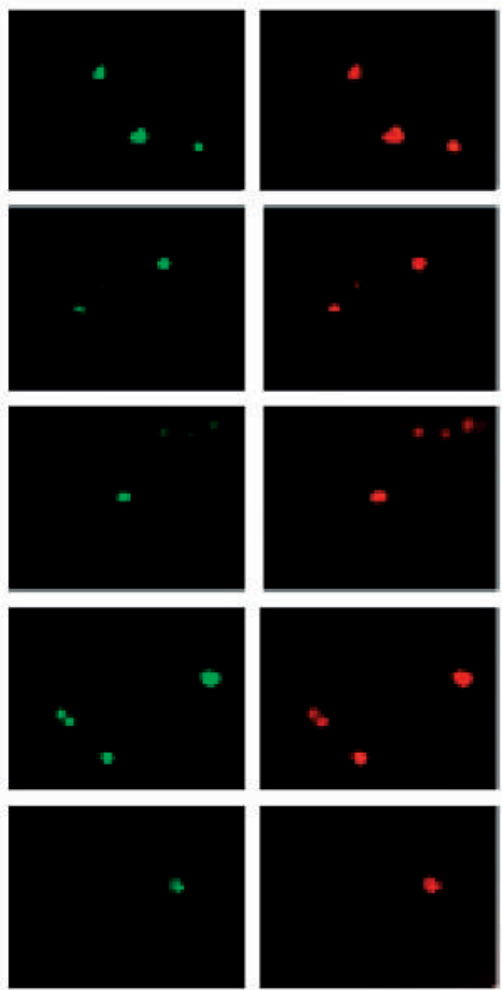

Cy3
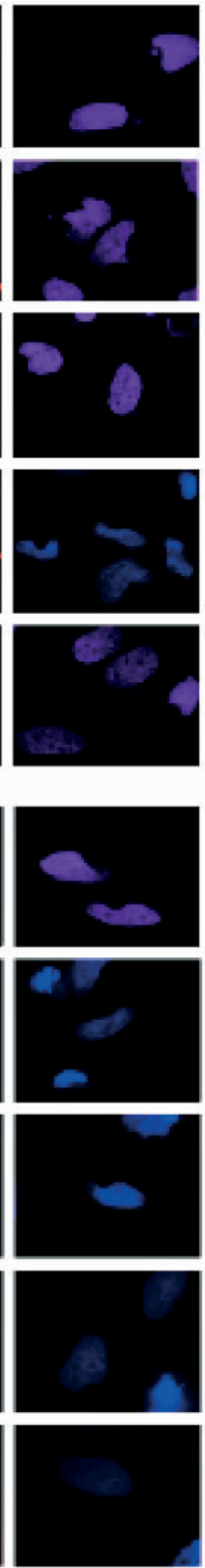

DAPI
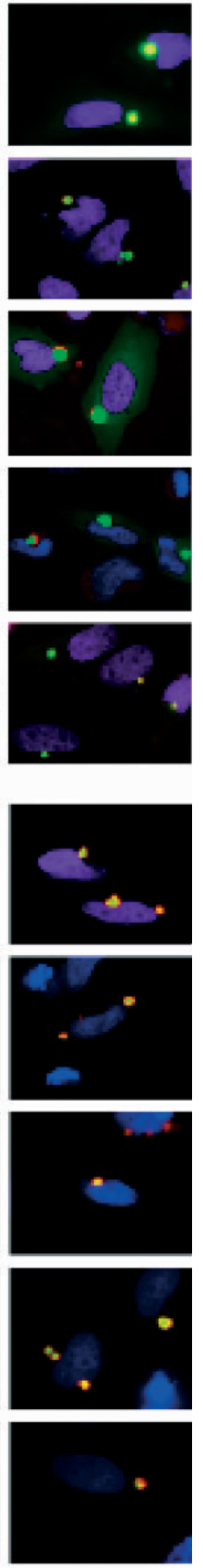

Merged
Figure 2 Co-localisation of ASC with wild type or mutated M/P-fl and -d2 in HeLa cells co-transfected with ASC-V5, and wild type or mutated pEGFP-fl (A) or pEGFP-MEFV-d2 (B) expression vectors. The $M / P$ protein was visualised directly with GFP fluorescence; ASC-V5 was revealed with an anti-V5 antibody followed by $\mathrm{Cy} 3$ tagged secondary antibody, and the nuclei were stained with DAPI. Merged images are shown on the right. The data shown are representative of several transient transfections of each construct. Cells expressing low levels of recombinant proteins are shown (see text). patterns, which resemble those reported in $\mathrm{CHO}$ cells overexpressing $\mathrm{M} / \mathrm{P}-\mathrm{fl}^{11}{ }^{11}$ most likely represent artefacts due to exaggerated overexpression of recombinant proteins. Similarly, when expressed at high levels, M/P-d2-GFP also resulted in the formation of cytoplasmic aggregates (fig 2B, M694V panel), an observation that further supports the hypothesis that overexpression of recombinant proteins may result in saturation of physiological protein transport pathways. ${ }^{16}$
The fact that the coexpression of wild type M/P-fl and ASC triggers the formation of specks that contain the two proteins $^{7}$ prompted us (a) to test the effect of FMF associated mutations on the formation of these cytoplasmic structures and $(b)$ to assess the effect of ASC on the cell compartmentalisation of both wild type M/P-d2, which when expressed alone is localised mainly in the nucleus, and several mutated M/P-d2 isoforms. To this end, we expressed 
ASC-V5 together with wild type or mutated M/P-GFP proteins. Although the interaction between $\mathrm{M} / \mathrm{P}-\mathrm{fl}$ and ASC has been shown to occur through their respective PYD domains, the possibility cannot be excluded that mutations located in the B30.2/SPRY domain (M680I, M694V, M694I, $\mathrm{V726A}$ ) or in exon 2 (El48Q) can alter the overall three dimensional conformational structure of $\mathrm{M} / \mathrm{P}$ and in turn lead to a lack of interaction with ASC. In addition, we tested the A89T (nt $265 \mathrm{G} \rightarrow \mathrm{A}$ ) mutation that we identified in one patient of Armenian ancestry who had FMF (unpublished data). This missense mutation is of particular interest because it is localised in the PYD domain of $\mathrm{M} / \mathrm{P}$; it could, therefore, directly interfere with the protein-protein interaction step documented between the two PYDs. As expected, speck positive cells were observed after co-expression of wild type M/P-fl with ASC (fig 2A); the specks, which were visualised in the cytoplasm of HeLa cells, contained both $\mathrm{M}$ / P-fl-GFP and ASC-V5, as attested to by the green and the red labelling, respectively. When each of the mutated M/P-fl proteins was expressed together with ASC (fig 2A; data not shown for the M694I and E148Q mutants), we still observed cytoplasmic specks in which both proteins colocalised. The morphology, number, and cell location of these specks were similar to those observed with the wild type M/P-fl (fig 2A).

We then tested the ability of M/P-d2 to form specks when co-expressed with ASC. As shown in fig 2B, the co-expression of ASC dramatically modifies the cell compartmentalisation of the M/P-d2 isoform, the localisation of which is mainly nuclear when expressed alone. The co-expression of the wild type $\mathrm{M} / \mathrm{P}-\mathrm{d} 2$ isoform and ASC did indeed result in the formation of cytoplasmic specks in which both proteins colocalised. In addition, speck positive cells did not display any green fluorescence in the nucleus, thereby indicating that the great majority of $\mathrm{M} / \mathrm{P}-\mathrm{d} 2$ was trapped in the specks. These results, therefore, strongly suggest that, like $\mathrm{M} / \mathrm{P}-\mathrm{fl}, \mathrm{M} / \mathrm{P}-\mathrm{d} 2$ is able to interact with ASC. In addition, as for $\mathrm{M} / \mathrm{P}$-fl, cells coexpressing mutant $\mathrm{M} / \mathrm{P}-\mathrm{d} 2$ proteins with ASC displayed a fluorescence pattern identical to that of cells expressing ASC with the wild type $\mathrm{M} / \mathrm{P}-\mathrm{d} 2$ isoform (fig $2 \mathrm{~B}$, and data not shown for the M694I mutant). In particular, there was no discernible M/P protein in the nucleus of speck positive cells.

Altogether, these data show that, at least in HeLa cells overexpressing the relevant constructs, the most frequent mutations found in patients with FMF (that is M694V, V726A, M680I, M694I, all of which are located in the B30.2/ SPRY domain of the protein), as well as the E148Q mutation, do not alter the subcellular localisation of the M/P protein, nor its interaction with ASC and the subsequent colocalisation of the two proteins in specks. In addition, the coexpression of ASC with the M/P-fl or - $\mathrm{d} 2$ isoform carrying the A89T mutation located within the PYD domain also resulted in the formation of specks in which both proteins colocalised. The latter result indicates that the A89 residue, which is conserved in rat and mouse $\mathrm{M} / \mathrm{P},{ }^{17}$ is not essential for the interaction between the two PYD domains of ASC and $\mathrm{M} / \mathrm{P}$, even though the mutation is adjacent to the $\mathrm{R} 88$ residue that is supposed to be involved in this interaction. ${ }^{7}$

We conclude, therefore, that the functional consequences of the FMF associated mutations are probably not related to a mislocalisation of the mutant $\mathrm{M} / \mathrm{P}-\mathrm{fl}$ and $-\mathrm{d} 2$ isoforms. In addition, these data show that these mutations do not alter the ASC induced subcellular redistribution of M/P-fl and -d2, each isoform still being able to participate in speck formation. The situation may, however, be different in human neutrophils and macrophages where M/P is normally expressed. Furthermore, as FMF manifests by recurrent episodes of inflammation and as patients with FMF are symptom free between each crisis, it is not possible to exclude a mislocalisation of the mutant $\mathrm{M} / \mathrm{P}$ proteins expressed alone or in combination with ASC solely after induction of specific signalling pathway(s) by the factors triggering crises, which are still to be determined.

\section{ACKNOWLEDGEMENTS}

This study was supported by a grant from AFM/INSERM (Réseau de recherche sur les maladies rares). C Cazeneuve is the recipient of a fellowship from the Institut National de la Santé et de la Recherche Médicale, and S Papin is the recipient of a fellowship from the Société de Secours des Amis des Sciences.

\section{Authors' affiliations}

C Cazeneuve, S Papin, I Jéru, P Duquesnoy, S Amselem, Institut National de la Santé et de la Recherche Médicale (Unité 468), Hôpital Henri-Mondor, Créteil, France

The first two authors contributed equally to the work

Correspondence to: $\operatorname{Dr} \mathrm{S}$ Amselem, Service de Biochimie et de Génétique Moléculaire-INSERM U468, Hôpital Henri-Mondor, 51 Avenue du Maréchal de-Lattre-de-Tassigny, F-94010 Créteil, France; serge.amselem@im3.inserm.fr

Received 23 June 2003

Accepted 17 September 2003

\section{REFERENCES}

1 The French FMF Consortium. A candidate gene for familial Mediterranean fever. Nature Genet 1997;17:25-31.

2 The International FMF Consortium. Ancient missense mutations in a new member of the RoRet gene family are likely to cause familial Mediterranean fever. Cell 1997;90:797-807.

3 Sarrauste de Menthiere C, Terriere S, Pugnere D, Ruiz M, Demaille J, Touitou I. INFEVERS: the Registry for FMF and hereditary inflammatory disorders mutations. Nucleic Acids Res 2003;31:282-5

4 Notarnicola C, Manna R, Rey J, Touitou I. Y688X, the first nonsense mutation in familial Mediterranean fever (FMF). Hum Mutat 2001;17:79.

5 Bertin J, DiStefano P. The PYRIN domain: a novel motif found in apoptosis and inflammation proteins. Cell Death Differ 2000;7:1273-4.

6 Gumucio D, Diaz A, Schaner P, Richards N, Babcock C, Schaller M, Cesena T. Fire and ICE: the role of pyrin domain-containing proteins in inflammation and apoptosis. Clin Exp Rheumatol 2002;20(Suppl 26):S45-53.

7 Richards N, Schaner P, Diaz A, Stuckey J, Shelden E, Wadhwa A, Gumucio D. Interaction between pyrin and the apoptotic speck protein (ASC) modulates ASC-induced apoptosis. J Biol Chem 2001;276:39320-9.

8 Chae J, Komarow H, Cheng J, Wood G, Raben N, Liu P, Kastner D. Targeted disruption of pyrin, the FMF protein, causes heightened sensitivity to endotoxin and a defect in macrophage apoptosis. Mol Cell

2003;11:591-604.

9 Pawlowski K, Pio F, Chu Z, Reed J, Godzik A. PAAD, a new protein domain associated with apoptosis, cancer and autoimmune diseases. Trends Biochem Sci 2001;26:85-7.

10 Martinon F, Hofmann K, Tschopp J. The pyrin domain: a possible member of the death domain-fold family implicated in apoptosis and inflammation. Curr Biol 2001;11:R118-20.

11 Papin S, Duquesnoy P, Cazeneuve C, Pantel J, Coppey-Moisan M, Dargemont C, Amselem S. Alternative splicing at the MEFV locus involved in familial Mediterranean fever regulates translocation of the marenostrin/pyrin protein to the nucleus. Hum Mol Genet 2000;9:3001-9.

12 Diaz A, Richards N, Schaner P, Gumucio D. Expression of alternatively spliced forms of pyrin in human leukocytes and synovial fibroblasts. Clin Exp Rheumatol 2002;20(Suppl 26):S82.

13 Centola M, Wood G, Frucht DM, Galon J, Aringer M, Farrell C, Kingma DW, Horwitz ME, Mansfield E, Holland SM, O'Shea JJ, Rosenberg HF, Malech HL, Kastner DL. The gene for familial Mediterranean fever, MEFV, is expressed in early leukocyte development and is regulated in response to inflammatory mediators. Blood 2000;95:3223-31.

14 Chen X, Bykhovskaya Y, Tidow N, Hamon M, Bercovitz Z, Spirina O, FischelGhodsian N. The familial Mediterranean fever protein interacts and colocalizes with a putative Golgi transporter. Proc Soc Exp Biol Med 2000;224:32-40.

15 Conway K, McConnell B, Bowring C, Donald C, Warren S, Vertino P. TMS1, a novel proapoptotic caspase recruitment domain protein, is a target of methylation-induced gene silencing in human breast cancers. Cancer Res 2000;60:6236-42.

16 Kremmidiotis G, Lensink I, Bilton R, Woollatt E, Chataway T, Sutherland G, Callen D. The Batten disease gene product (CLN3p) is a Golgi integral membrane protein. Hum Mol Genet 1999;8:523-31.

17 Chae JJ, Centola M, Aksentijevich I, Dutra A, Tran M, Wood G, Nagaraju K, Kingma DW, Liu PP, Kastner DL. Isolation, genomic organization, and expression analysis of the mouse and rat homologs of MEFV, the gene for familial mediterranean fever. Mamm Genome 2000;1 1:428-35. 